\title{
Article \\ Gene Expression Changes of Humans with Primary Mitral Regurgitation and Reduced Left Ventricular Ejection Fraction
}

\author{
Feng-Chun Tsai ${ }^{1,2}$, Yu-Lin Chen ${ }^{3}$, Kun-Chi Yen ${ }^{2,4}$, Cheng-Hsun Chiu ${ }^{5,6}$, Jui-Hsuan Chen ${ }^{3}$, Yung-Hsin Yeh ${ }^{2,4}$ \\ and Pei-Chien Tsai $3,5,7,8, *$
}

Citation: Tsai, F.-C.; Chen, Y.-L.; Yen, K.-C.; Chiu, C.-H.; Chen, J.-H.; Yeh, Y.-H.; Tsai, P.-C. Gene Expression Changes of Humans with Primary Mitral Regurgitation and Reduced Left Ventricular Ejection Fraction. Int. J. Mol. Sci. 2021, 22, 3454. https:// doi.org/10.3390/ijms22073454

Academic Editor: Ralph Knöll

Received: 7 March 2021

Accepted: 25 March 2021

Published: 26 March 2021

Publisher's Note: MDPI stays neutral with regard to jurisdictional claims in published maps and institutional affiliations.

Copyright: (c) 2021 by the authors. Licensee MDPI, Basel, Switzerland. This article is an open access article distributed under the terms and conditions of the Creative Commons Attribution (CC BY) license (https:// creativecommons.org/licenses/by/ $4.0 /)$.
1 Division of Cardiac Surgery, Chang Gung Memorial Hospital, Taoyuan City 333, Taiwan; lutony@cgmh.org.tw

2 School of Medicine, Chang Gung University, Taoyuan City 33302, Taiwan; dcsam999@gmail.com (K.-C.Y.); yys0tw@yahoo.ca (Y.-H.Y.)

3 Department of Biomedical Sciences, Chang Gung University, Taoyuan City 33302, Taiwan; cenachen46@gmail.com (Y.-L.C.); qswe81008@gmail.com (J.-H.C.)

4 Cardiovascular Department, Chang Gung Memorial Hospital, Taoyuan City 333, Taiwan

5 Division of Pediatric Infectious Diseases, Department of Pediatrics, Chang Gung Memorial Hospital, Taoyuan City 333, Taiwan; chchiu@adm.cgmh.org.tw

6 Molecular Infectious Disease Research Center, Chang Gung Memorial Hospital, Taoyuan City 333, Taiwan

7 Graduate Institute of Biomedical Sciences, Chang Gung University, Taoyuan City 33302, Taiwan

8 Healthy Aging Research Center, Chang Gung University, Taoyuan City 33302, Taiwan

* Correspondence: pctsai@gap.cgu.edu.tw; Tel.: +886-3-2118800 (ext. 3857)

Abstract: Patients with primary mitral regurgitation (MR) may remain asymptomatic for many years. For unknown reasons, some shift from a compensated to a decompensated state and progress to fatal heart failure. To elucidate the genetic determinants of this process, we recruited 28 patients who underwent mitral valve surgery and stratified them into control, compensated MR, and decompensated MR groups. Tissue biopsies were obtained from the patients' left ventricular (LV) lateral wall for a transcriptome-wide profiling of 64,769 probes to identify differentially expressed genes (DEGs). Using cutoff values at the $1 \%$ FDR significance level and sex- and age-adjusted regression models, we identified 12 significant DEGs (CTGF, MAP1B, SERPINE1, MYH9, MICAL2, MYO1D, CRY1, AQP7P3, HTRA1, PRSS23, IGFBP2, and FN1). The most significant gene was CTGF (adjusted $\left.\mathrm{R}^{2}=0.74, p=1.80 \times 10^{-8}\right)$. We found that the majority of genes expressed in the more advanced decompensated MR group were pro-fibrotic genes associated with cardiac fibrosis. In particular, six pro-fibrotic genes (CTGF, SERPINE1, MYH9, HTRA1, PRSS23, and FN1) were overexpressed and enriched in pathways involved in ECM (extracellular matrix) protein remodeling. Therapeutic interventions that antagonize these six genes may slow the progression toward decompensated MR.

Keywords: mitral regurgitation; cardiac fibrosis; reduced ejection fraction; transcriptome-wide association analysis

\section{Introduction}

Primary mitral regurgitation (MR) is an abnormality of the mitral valve, most commonly due to myxomatous degeneration of the valve leaflets [1]. This prevents the valve from closing completely, resulting in retrograde blood flow from the left ventricle into the left atrium. During the compensatory phase of the disease, the LV dilates to maintain normal wall stress and pressure [1]. As the disease progresses, the ventricle exhibits more spherical shape, further increasing the LV end-diastolic pressure. At the same time, the contractile state of the myocardium decreases under the combined effect of myofiber reduction and interstitial fibrosis. The decompensated phase of the disease occurs when irreversible LV dysfunction develops with symptoms of heart failure [1].

Little is known about the molecular mechanisms that cause irreversible LV dysfunction in decompensated MR. Only recently, gene expression has been compared between 
individuals with compensated and decompensated MR using a panel of 109 candidate genes selected from animal and human studies [2]. The transition to decompensated MR is characterized by increased expression of $N P P A, N P P B$, inflammatory, ECM regulatory, and apoptotic genes, as well as aberrant expression of calcium regulatory and mitochondrial function genes [2].

The aim of this study was to perform whole transcriptome profiling of patients with compensated and decompensated MR. Subsequently, differentially expressed genes (DEGs) were analyzed by Gene Ontology (GO) and Kyoto Encyclopedia of Genes and Genomes (KEGG) analysis to identify pathological pathways in patients with compensated and decompensated MR.

\section{Results}

\subsection{Study Participants}

The baseline characteristics of the 28 patients are shown in Table 1. Patients were divided into three groups: control, compensated MR group, and decompensated MR group. In the analysis of LV dilatation, the control and CMR groups were combined and analyzed because there were significant differences between the control and CMR groups in LV end-diastolic diameter (LVEDD) and LV end-systolic diameter (LVESD), which were larger in the CMR group than in the control group $\left(p=5.86 \times 10^{-4}\right.$ and $p=0.049$, respectively), but there were no significant differences in shortening fraction (SF) or ejection fraction (EF) between the two groups. The DMR group was not included in the analysis of LV dilatation because of the significantly lower EF in the DMR group. In contrast, SF and EF were significantly lower in the DMR group than in the control and CMR groups $\left(p=3.30 \times 10^{-4}\right.$ and $2.49 \times 10^{-5}$, respectively), whereas LVEDD and LVESD were not significantly different from the other two groups (except for LVESD in DMR versus CMR, $p=0.024)$. In the analysis of $\mathrm{LV}$ dysfunction due to reduced EF, the DMR, CMR, and control groups were combined.

Table 1. Clinical characteristics of 28 patients referred for mitral valve correction surgery.

\begin{tabular}{|c|c|c|c|c|c|c|}
\hline $\begin{array}{l}\text { Clinical } \\
\text { Variable }\end{array}$ & $\begin{array}{c}\text { Control } \\
n=11\end{array}$ & $\begin{array}{l}\text { CMR } \\
n=13\end{array}$ & $\begin{array}{l}\text { DMR } \\
n=4\end{array}$ & $\begin{array}{c}p \text { Value } \\
\text { (CMR vs. Control) }\end{array}$ & $\begin{array}{c}p \text { Value } \\
\text { (DMR vs. Control) }\end{array}$ & $\begin{array}{c}p \text { Value } \\
\text { (DMR vs. CMR) }\end{array}$ \\
\hline Age, years & $62.45 \pm 11.34$ & $59.46 \pm 12.87$ & $59.75 \pm 13.96$ & 0.551 & 0.555 & 0.970 \\
\hline \multicolumn{7}{|l|}{$\operatorname{Sex}(n, \%)$} \\
\hline Female & $7(64)$ & $3(23)$ & $2(50)$ & 0.0953 & 0.999 & 0.5378 \\
\hline Male & $4(36)$ & $10(77)$ & $2(50)$ & & & \\
\hline \multicolumn{7}{|c|}{ Parameters for LV diameter } \\
\hline LVEDD, $\mathrm{mm}$ & $47.27 \pm 4.03$ & $57.85 \pm 8.07$ & $61.25 \pm 14.93$ & $5.86 \times 10^{-4}$ & 0.157 & 0.554 \\
\hline LVESD, mm & $31.18 \pm 4.94$ & $37.69 \pm 9.27$ & $54.00 \pm 17.38$ & 0.049 & 0.077 & 0.024 \\
\hline \multicolumn{7}{|c|}{ Parameters for LV function } \\
\hline $\mathrm{SF}, \%$ & $0.34 \pm 0.07$ & $0.35 \pm 0.10$ & $0.13 \pm 0.10$ & 0.767 & $3.30 \times 10^{-4}$ & $1.20 \times 10^{-3}$ \\
\hline $\mathrm{EF}, \%$ & $62.27 \pm 9.01$ & $64.46 \pm 12.69$ & $28.75 \pm 9.07$ & 0.637 & $2.49 \times 10^{-5}$ & $1.12 \times 10^{-4}$ \\
\hline
\end{tabular}

Values are mean $\pm \mathrm{SD}$ in all variables except for gender; $n$, individuals; CMR, compensated MR; DMR, decompensated MR; LVEDD, left ventricular end diastolic diameter; LVESD, left ventricular end systolic diameter; SF, shortening fraction; EF, ejection fraction. $p$-Values for continuous variables were calculated by Student's $t$-test followed F test of equality of variances; $p$-Values for gender differences were calculated by Fisher's exact test.

\subsection{Identification of DEGs Associated with LV Dilatation and LV Dysfunction}

A transcriptome-wide association analysis was performed on 64,769 probes covering 25,100 annotated human genes, adjusted for sex and age as covariates (Figure 1). The first and second principal components (PCs) jointly explained $34.3 \%$ of the total variance. Compensated MR and controls were similar to each other on PC1 and PC2, while decompensated MR was separate from compensated MR and controls on PC2. 


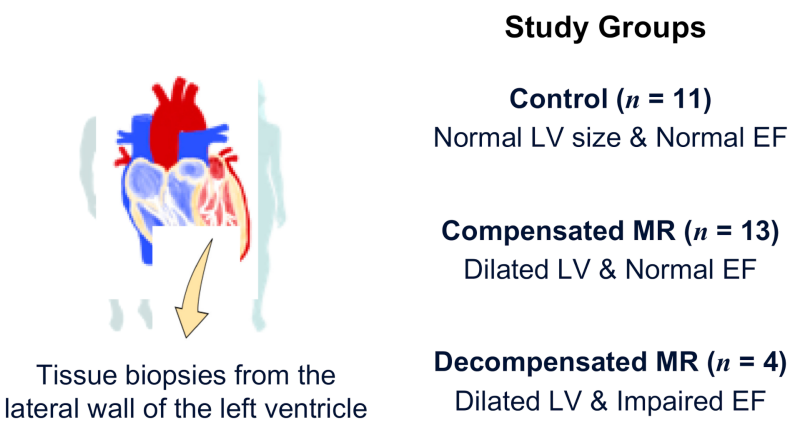

\section{Transcriptome-wide association analysis (using 64,769 probes)}

Gene expression LV Parameter + Sex + Age LVESD, LVEDD

Control
+ CMR
Passed 1\% FDR
significance level
$\downarrow$
$\mathbf{0 ~ D E G}$

EF

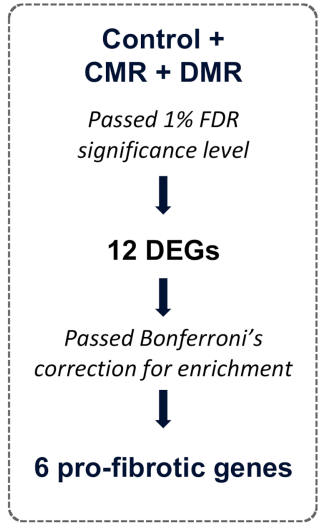

Principal Component Analysis (using 64,769 probes)

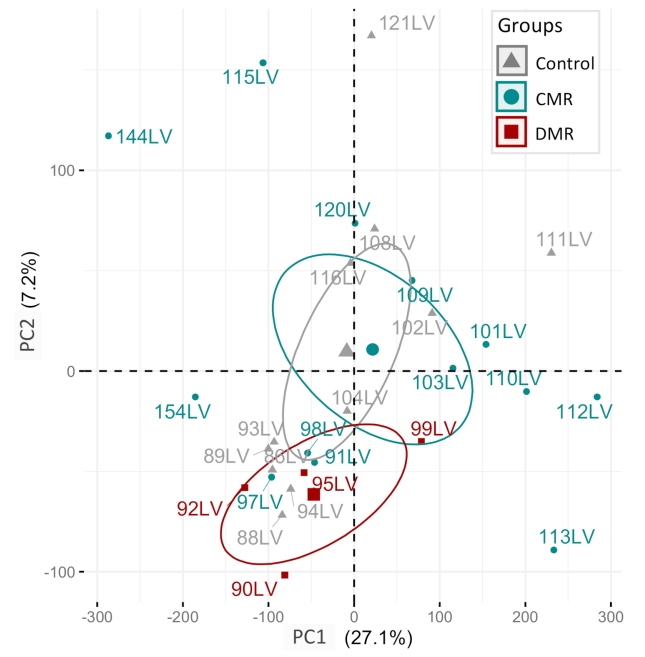

Figure 1. Summary of workflow and main findings.

When using the LVEDD and LVESD parameters to compare gene expression changes in patients with normal and dilated LV, we did not find DEGs that passed the $1 \%$ FDR significance level (Figure 2A,B). However, after combining the three groups, 19 probes passed the 1\% FDR significance level and were associated with reduced EF (Figure 2C). Four probes were positively correlated (i.e., positive $\beta$ coefficients) and 15 probes were negatively correlated. Of these probes, 12 significant DEGs were located in the coding region of the transcribed genes, one of which was positively correlated with EF and the other 11 were negatively correlated with EF.

Table 2 shows the the top suggestive DEGs associated with increased LVEDD and LVESD including probe set IDs, chromosomes, start and end base pairs (GRCh37/hg19), symbols of transcribed genes and their descriptions, followed by the 12 significant DEGs associated with reduced EF.

Figure 3 shows the direction of the $\beta$ coefficient correlation values for each of the 12 significant DEGs. The expression levels of all genes increased with reduced EF, and only the expression of $A Q P 7 P 3$ decreased with reduced $\mathrm{EF}$. 
A $\operatorname{LVEDD}($ Control + CMR)

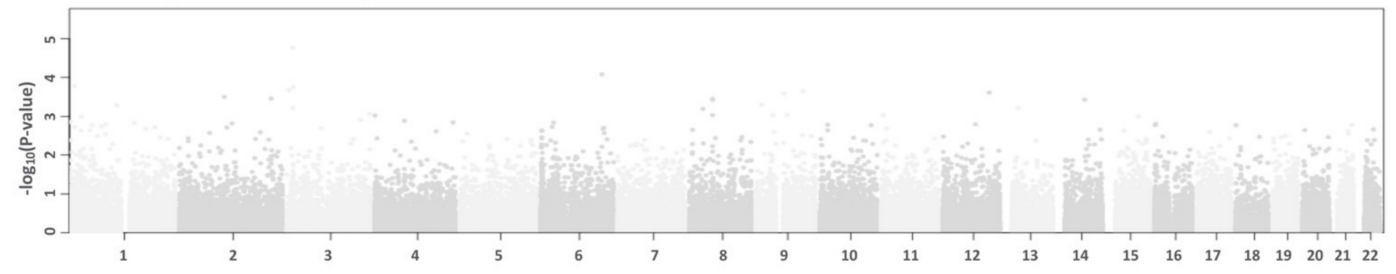

B LVESD (Control + CMR)
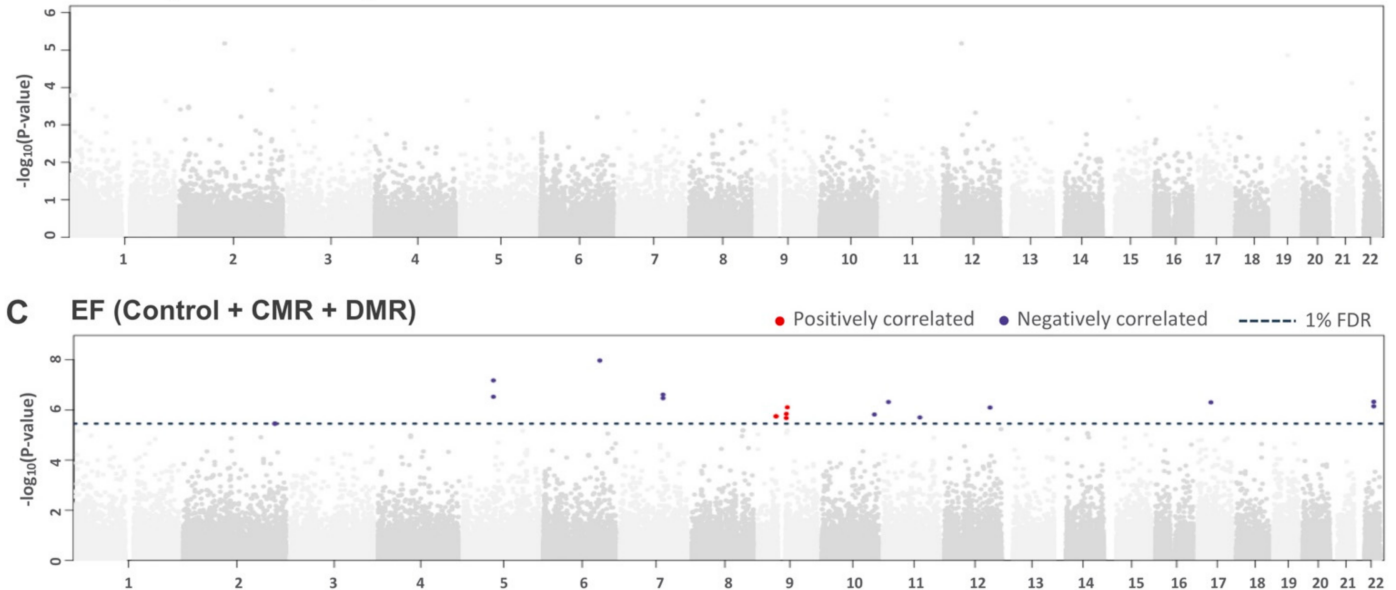

Figure 2. Transcriptome-wide association analysis. (A) LVEDD, (B) LVESD, (C) Ejection fraction. Each data point on the Manhattan plot corresponds to a TWAS result of $-\log _{10}$ of the $p$-value. The blue dashed line corresponds to the transcriptome-wide significance threshold for $1 \%$ FDR, and for ejection fraction, the significance threshold is at $p=3.56 \times 10^{-6}$. Positive correlation probes are in red and negative correlation probes are in blue.
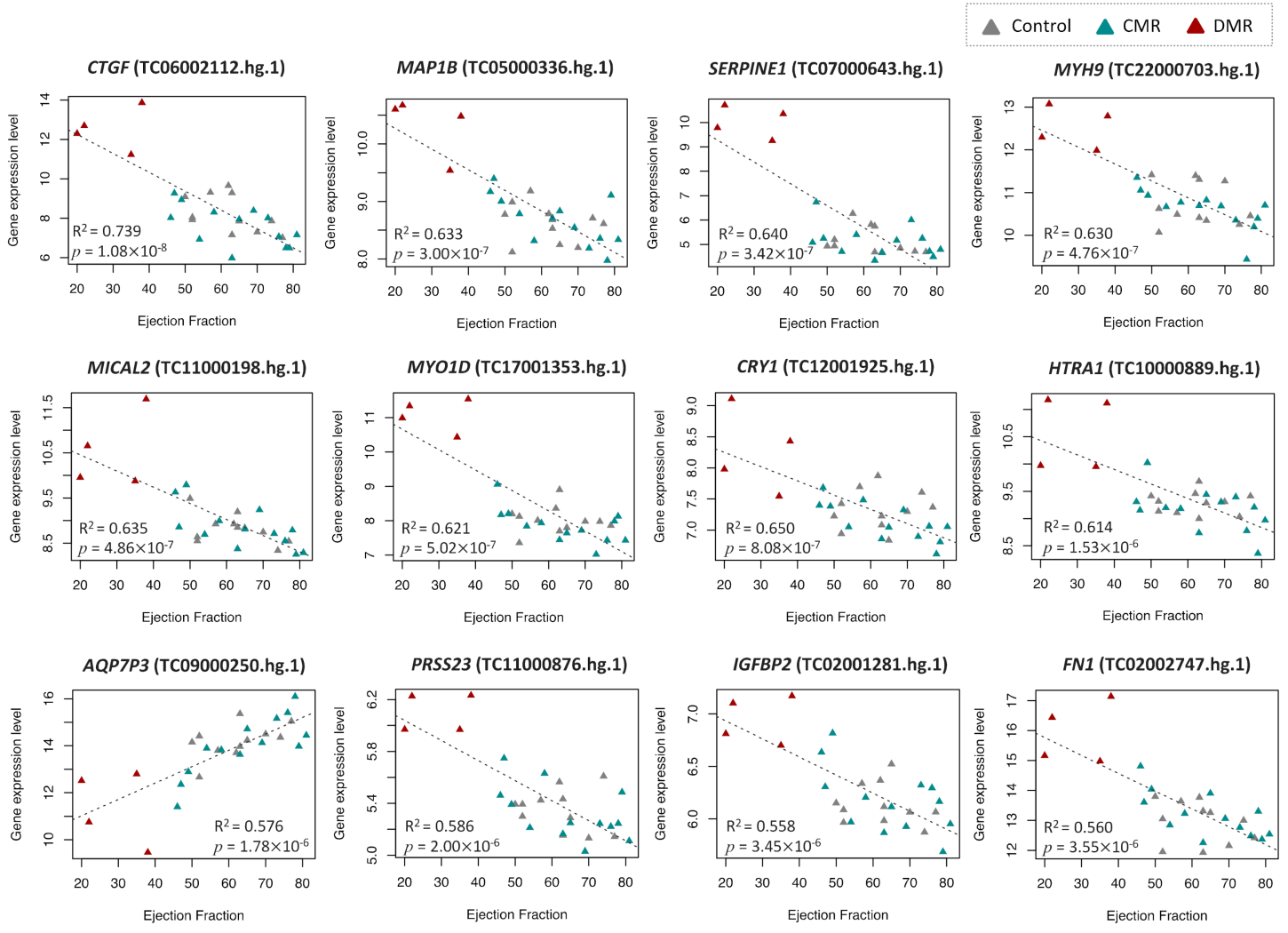

Figure 3. Correlation of gene expression levels with reduced EF in each of the 12 significant DEGs. 
Table 2. Top DEGs associated with LVEDD, LVESD, and EF parameters.

\begin{tabular}{|c|c|c|c|c|c|c|c|c|}
\hline Probe Set ID & CHR & Start & End & $\beta$ Coef. & S.E. & $p$ Value & Gene Symbol & Description \\
\hline \multicolumn{9}{|l|}{ LVEDD } \\
\hline TC03001196.hg.1 & 3 & 14389951 & 14394068 & 0.025 & 0.004 & $1.70 \times 10^{-5}$ & LINC01267 & $\begin{array}{l}\text { long intergenic non-protein } \\
\text { coding RNA } 1267\end{array}$ \\
\hline $\begin{array}{l}\text { TC01002205.hg. } 1 \\
\text { LVESD }\end{array}$ & 1 & 11917521 & 11918992 & 0.298 & 0.065 & $1.68 \times 10^{-4}$ & $N P P B$ & $\begin{array}{l}\text { natriuretic peptide } \\
\text { precursor B }\end{array}$ \\
\hline TC02002167.hg.1 & 2 & 105974169 & 106055230 & -0.037 & 0.006 & $6.67 \times 10^{-6}$ & FHL2 & $\begin{array}{l}\text { four and a half LIM } \\
\text { domains } 2\end{array}$ \\
\hline TC03001196.hg.1 & 3 & 14389951 & 14394068 & 0.025 & 0.004 & $1.01 \times 10^{-5}$ & LINC01267 & $\begin{array}{l}\text { long intergenic non-protein } \\
\text { coding RNA } 1267\end{array}$ \\
\hline \multicolumn{9}{|r|}{ 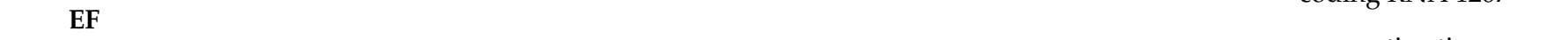 } \\
\hline TC06002112.hg.1 & 6 & 132269316 & 132272518 & -0.100 & 0.012 & $1.80 \times 10^{-8}$ & CTGF & $\begin{array}{l}\text { connective tissue } \\
\text { growth factor }\end{array}$ \\
\hline TC05000336.hg.1 & 5 & 71403061 & 71505397 & -0.036 & 0.005 & $3.00 \times 10^{-7}$ & $M A P 1 B$ & $\begin{array}{l}\text { microtubule associated } \\
\text { protein } 1 \mathrm{~B}\end{array}$ \\
\hline TC07000643.hg.1 & 7 & 100770370 & 100782547 & -0.092 & 0.013 & $3.43 \times 10^{-7}$ & SERPINE1 & $\begin{array}{c}\text { serpin peptidase inhibitor, } \\
\text { clade E (nexin, } \\
\text { plasminogen activator } \\
\text { inhibitor type } 1 \text { ), member } 1\end{array}$ \\
\hline TC22000703.hg.1 & 22 & 36677323 & 36784063 & -0.040 & 0.006 & $4.76 \times 10^{-7}$ & MYH9 & $\begin{array}{c}\text { myosin, heavy chain } 9, \\
\text { non-muscle } \\
\text { microtubule associated }\end{array}$ \\
\hline TC11000198.hg.1 & 11 & 12115543 & 12285332 & -0.038 & 0.006 & $4.86 \times 10^{-7}$ & MICAL2 & $\begin{array}{l}\text { monooxygenase, calponin } \\
\text { and LIM domain } \\
\text { containing } 2\end{array}$ \\
\hline TC17001353.hg.1 & 17 & 30819627 & 31203902 & -0.061 & 0.009 & $5.20 \times 10^{-7}$ & MYO1D & myosin ID \\
\hline TC12001925.hg.1 & 12 & 107385142 & 107487607 & -0.025 & 0.004 & $8.08 \times 10^{-7}$ & CRY1 & cryptochrome circadian \\
\hline TC10000889.hg.1 & 10 & 124221041 & 124274424 & -0.029 & 0.005 & $1.53 \times 10^{-6}$ & HTRA1 & HtrA serine peptidase 1 \\
\hline TC09000250.hg.1 & 9 & 42858152 & 42893137 & 0.072 & 0.011 & $1.78 \times 10^{-6}$ & AQP7P3 & aquaporin 7 pseudogene 3 \\
\hline TC11000876.hg.1 & 11 & 86511282 & 86663886 & -0.016 & 0.003 & $2.00 \times 10^{-6}$ & PRSS23 & protease, serine, 23 \\
\hline TC02001281.hg.1 & 2 & 217497551 & 217529159 & -0.018 & 0.003 & $3.54 \times 10^{-6}$ & IGFBP2 & $\begin{array}{l}\text { insulin like growth factor } \\
\text { binding protein } 2\end{array}$ \\
\hline TC02002747.hg.1 & 2 & 216225163 & 216300895 & -0.060 & 0.010 & $3.55 \times 10^{-6}$ & FN1 & fibronectin 1 \\
\hline
\end{tabular}

CHR, chromosome; $\beta$ coef., $\beta$ coefficients; S.E., standard error. P-value were evaluated by linear regression model adjusted for age and gender.

\subsection{Functional and Pathway Enrichment Analysis}

Enrichment of GO and KEGG pathways was performed for the 12 significant DEGs. Figure $4 \mathrm{~A}$ shows that for the GO.BP, the relevant terms were extracellular structure organization (GO:0043062), extracellular matrix organization (GO:0030198), and integrinmediated signaling pathway (GO:0007229). For the GO.CC, they were collagen-containing extracellular matrix (GO:0062023). For the GO.MF, they were actin binding (GO:0003779), integrin binding (GO:0008305), growth factor binding (GO:0019838), and insulin-like growth factor binding (GO:0031994). For the KEGG pathway, they were the hippo signaling pathway (hsa04390), apelin signaling pathways (hsa04371), and AGE-RAGE signaling pathway in diabetic complications (hsa04933).

Figure $4 \mathrm{~B}$ is a conceptual network diagram of genes showing the relationship between mRNA and enrichment pathways. Examples of the most frequently co-occurring genes lying in the same pathways were CTGF/SERPINE1 and MHY9/MYO1D. Figure 4C shows the STRING network using 11 genes ( $A Q P 7 P 3$ was omitted as it was not searchable in the STRING database). The network revealed that the highest interaction enrichment was between six genes. 
A

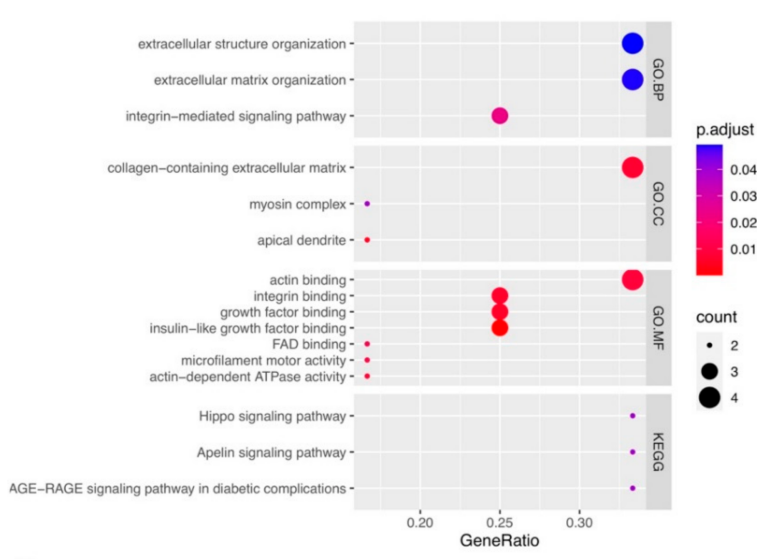

C

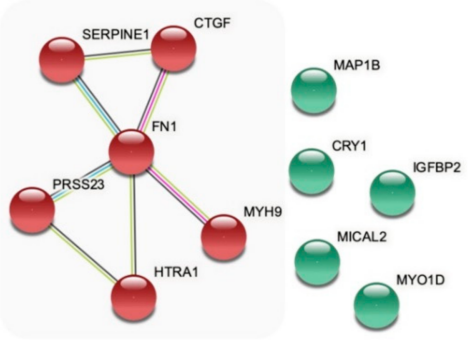

Go.cC
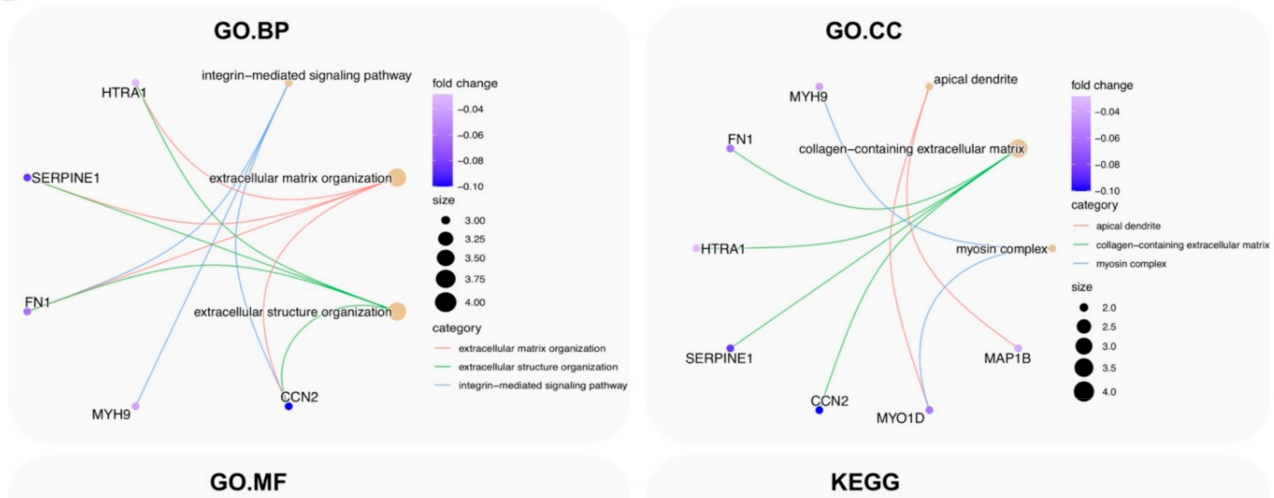

KEGG

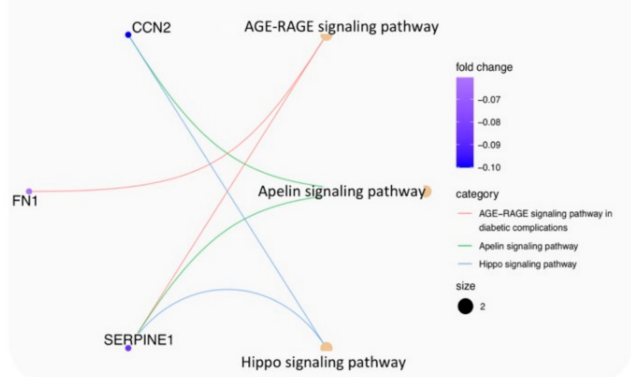

Figure 4. Enriched GO terms and KEGG pathways for the 12 significant DEGs. (A) GO terms and pathways that passed Bonferroni correction $p<0.05$ and $q<0.05$. (B) Relationships between mRNA and GO terms in BP (biological process), CC (cellular component), MF (molecular function), and KEGG pathways; fold change are $\beta$ coefficient correlation values (C) STRING network showing the highest interaction enrichment between CTGF, SERPINE1, FN1, PRSS23, HTRA1, and MYH9 genes (PPI enrichment $p$ value $1.8 \times 10^{-4}$ ).

\section{Discussion}

Our transcriptome-wide profiling revealed that the majority of genes expressed during the transition to decompensated MR are pro-fibrotic genes. Cardiac fibrosis in the context of valvular disease refers to the proliferation of interstitial fibrosis involving the deposition of collagen-rich ECM in the interstitial space between cells in response to abnormal pressure loads [3]. Short-term interstitial fibrosis can be adaptive, but sustained activation of fibrotic pathways causes excessive accumulation of ECM and disruption of tissue function. The compensated MR group showed only suggestive changes in gene expression compared to the control group. Regarding the top genes associated with LV dilatation, we observed decreased expression of FHL2, whose overexpression increases ECM production and interstitial fibrosis [4], and increased expression of $N P P B$, which has been shown to be a cardiomyocyte-derived antifibrotic factor in ventricular remodeling [5]. Increased 
expression of LINC01276, a non-coding RNA gene, which has not been previously reported with increased LV dilatation, was also observed.

For LV dysfunction-related decompensated MR, 12 significant DEGs (CTGF, MAP1B, SERPINE1, MYH9, MICAL2, MYO1D, CRY1, AQP7P3, HTRA1, PRSS23, IGFBP2 and FN1) passed the $1 \%$ FDR significance threshold. CTGF (encoding the CCN2 protein) was the most significantly transcribed gene in this study $\left(p=1.80 \times 10^{-8}\right)$. It connects with other genes involved in extracellular structural organization, ECM organization, integrin-mediated pathway, collagen-containing extracellular matrix, integrin binding, growth factor binding, insulin-like growth factor binding, Apelin signaling pathway and Hippo signaling pathway. CTGF is a well-known central mediator of fibrosis that activates myofibroblast and ECM protein remodeling. It is also significantly expressed in human myxomatous mitral valves compared to non-myxomatous mitral valves [6].

Five other pro-fibrotic genes in the STRING network are associated with CTGF. They are SERPINE1 that encodes PAI (plasminogen activator inhibitor)-1, whose loss-of-function mutations are associated with human cardiac fibrosis [7]. FN1 participates in ECM remodeling and its inhibition attenuates fibrosis and improves heart failure [8]. HTRA1 reduces cellular secretion of mature TGF- $\beta 1$, and CTGF synergizes with TGF- $\beta$ to induce ECM deposition [9]. PRSS23 is an activator of endothelial-to-mesenchymal transition and contributes to increased ECM protein production by cardiac fibroblasts, so exacerbating cardiac fibrosis [10]. MYH9 inhibition suppresses TGF- $\beta 1$-induced differentiation of lung fibroblast to myofibroblast [11].

TGF- $\beta$ also plays a crucial role in fibrosis and is released in the pressure-overloaded heart in addition to neurohumoral mediators such as angiotensin, aldosterone, and norepinephrine [12]. The TGF- $\beta$ pathway is controlled by ubiquitin-modifying enzymes and dysregulated ubiquitination is implicated in the deterioration of LV function [13]. So far, antagonizing TGF- $\beta$ in heart failure has been toxic to humans [3]. However, treatment of mice with monoclonal antibodies to CTGF revealed encouraging results with reduced LV mass, cardiomyocyte hypertrophy, and myocardial fibrosis [14]. We suggest that the pro-fibrotic genes identified in this study have the potential to be therapeutic targets.

The remaining genes in the STRING network are associated with decompensated $\mathrm{MR}$, but may not be directly related to fibrosis. They include $M A P 1 B$, which regulates the organization of the cytoskeleton [15]. MICAL2 affects the regeneration of myofilaments and muscle tissue [16]. MYO1D is expressed in podocytes [17]. CRY1 is a human circadian clock gene; and acute myocardial infarction and arrythmias are regulated by circadian clock genes [18]. AQP7P3 is the main aquaglyceroporin in the heart situated in between the capillary lumen and interstitial space that acts as a glycerol facilitator, and its dysfunction can lead to cardiac hypertrophy and death [19]. Finally, elevated IGFBP2 inhibits IGF-1, which regulates LV dysfunction and is a biomarker for predicting heart failure [20].

The present study has several limitations. First, it is a cross-sectional study that precludes a causal relationship. Second, MR is a progressive disease, and LV remodeling becomes advanced when the duration of MR is prolonged. We did not provide detailed information on disease duration and severity, because our review of patient records revealed that most patients had undergone mitral valve surgery for less than six months upon the diagnosis of severe MR. Some of the patients were referred from other hospitals, which resulted in a missing or slightly unreliable assessment of the duration of severe MR in the referred patients. In addition, the time point between moderate and severe MR may be defined rather arbitrarily as MR progresses over time. We think that for some patients, $\mathrm{LV}$ remodeling may be already underway when cardiac echography shows that the LV is already dilated, even if MR is still in the moderate phase. Considering the heterogeneous course of progression of MR-induced LV remodeling and the missing or unreliable documentation of the diagnosis of severe MR, we think that the duration of MR does not affect the results of this study. 


\section{Materials and Methods}

\subsection{Data Collection}

We recruited 28 unrelated patients; 11 of whom had normal LV diameter and preserved ejection fraction, most of whom had rheumatic disease with mitral stenosis; 13 subjects had primary MR with dilated LV and preserved ejection fraction; and 4 subjects had dilated $\mathrm{LV}$ with reduced ejection fraction. The study subjects were recruited between December 2013 and December 2018 at Chang Gung Memorial Hospital in Linkou, Taiwan. The study protocol complied with the ethical guidelines of the 1975 Declaration of Helsinki and was approved by the Chang-Gung Medical Foundation Institutional Review Board (IRB No: 20170824B0, approved on 14 June 2017; 100-4076A3, approved on 31 January 2012; 103-7141C, approved on 13 January 2015). Written informed consent was obtained from all participants. All patients underwent mitral valve correction surgery during which LV tissue was collected from the lateral endocardial wall. We excluded from this study patients with recent myocardial infarction, stroke, acute heart failure, and major valvular heart disease. Detailed baseline information, such as age, gender, disease history, previous Maze procedure history, rheumatic status and clinical measurements (LVEDD, LVESD, SF, EF) were collected from their electronic hospital records.

\subsection{RNA Isolation and Microarrays}

RNA samples were extracted from fresh LV tissue after cardiac surgery. Total RNA was extracted using Trizol reagent (Invitrogen, Carlsbad, CA, USA), purified and concentrated with WelPrep tissue RNA kit (Welgene, Taipei, Taiwan). RNA quality was assessed using an Agilent Bioanalyzer 2100 (Agilent Technologies, Santa Clara, CA, USA). Gene expression profiles in tissue RNA were analyzed using the human U133A (Clariom D) GeneChip (Affymetrix, Santa Clara, CA, USA) according to the manufacturer's protocol. Expression data were evaluated by Gene Level-SST RMA of Applied Biosystems Transcriptome Analysis Console (TAC).

\subsection{Statistical Analysis}

Clinical characteristics between two independent groups were compared using Student's $t$-test. Sex ratios were calculated using Fisher's exact test. Principal component analysis was used to identify potential covariates to be included in statistical models for analysis and to demonstrate clustering of patients. Transcriptome-wide association analysis was performed for each probe using a linear regression model, adjusting for age and sex. A false discovery rate (FDR) of $1 \%$ was set as the significance threshold using the BenjaminiHochberg method in R package qvalue [21]. GO and KEGG enrichment of DEGs was performed using hypergeometric analysis and Bonferroni correction with $p<0.05$ set as the significance level in R package clusterProfiler [22]. STRING v11.0 http:/ / string-db.org (accessed in 7 March 2021) [23].

\section{Conclusions}

We identified the molecular components in decompensated MR as being due to the overexpression of pro-fibrotic genes. Further animal models and human cardiomyocyte cultures are needed to validate their effects on cardiac fibrosis.

Author Contributions: Conceptualization, Y.-H.Y., P.-C.T. and F.-C.T.; methodology, P.-C.T.; formal analysis, P.-C.T., Y.-L.C. and J.-H.C.; resources, Y.-H.Y. and F.-C.T.; data curation, all authors; writingoriginal draft preparation, P.-C.T., Y.-L.C., Y.-H.Y., K.-C.Y., C.-H.C. and F.-C.T.; writing-review and editing, P.-C.T., Y.-L.C., Y.-H.Y. and F.-C.T.; visualization, P.-C.T., Y.-L.C. and J.-H.C.; supervision, Y.-H.Y., P.-C.T. and F.-C.T.; funding acquisition, Y.-H.Y., P.-C.T. and F.-C.T. All authors have read and agreed to the published version of the manuscript. 
Funding: F.-C. Tsai was funded by Chang Gung Memorial Hospital, grant number CMRPG3F2061/ CMRPG 3G1391; Y.-H. Yeh was funded by Chang Gung Memorial Hospital, grant number CORPG3J02913/ CMRPG3G1371-3; P.-C. Tsai was funded by Chang Gung Memorial Hospital, grant number CMRPD1J0082 / Ministry of Science and Technology, Taiwan, grant number NMRPD1K0941 and NZRPD1K0011.

Institutional Review Board Statement: This study protocol complies with the ethical guidelines of the 1975 Declaration of Helsinki and was approved by the Chang Gung Medical Foundation Institutional Review Board (IRB no: 20170824B0, 100-4076A3, 103-7141C).

Informed Consent Statement: Written informed consent was obtained from all subjects participating in the study.

Data Availability Statement: The data presented in this study are available from the corresponding authors upon request.

Conflicts of Interest: The authors declare no conflict of interest.

$\begin{array}{ll}\text { Abbreviations } \\ \text { FDR } & \text { False discovery rate } \\ \text { LV } & \text { Left ventricle } \\ \text { MR } & \text { Mitral regurgitation } \\ \text { ECM } & \text { Extracellular matrix } \\ \text { LVEDD } & \text { Left ventricular end-diastolic diameter } \\ \text { LVESD } & \text { Left ventricular end-systolic diameter } \\ \text { SF } & \text { Shortening fraction } \\ \text { EF } & \text { Ejection fraction }\end{array}$

\section{References}

1. El Sabbagh, A.; Reddy, Y.N.V.; Nishimura, R.A. Mitral Valve Regurgitation in the Contemporary Era: Insights Into Diagnosis, Management, and Future Directions. JACC Cardiovasc. Imaging 2018, 11, 628-643. [CrossRef]

2. McCutcheon, K.; Dickens, C.; van Pelt, J.; Dix-Peek, T.; Grinter, S.; McCutcheon, L.; Patel, A.; Hale, M.; Tsabedze, N.; Vachiat, A.; et al. Dynamic Changes in the Molecular Signature of Adverse Left Ventricular Remodeling in Patients With Compensated and Decompensated Chronic Primary Mitral Regurgitation. Circ. Heart Fail. 2019, 12, e005974. [CrossRef]

3. Sweeney, M.; Corden, B.; Cook, S.A. Targeting cardiac fibrosis in heart failure with preserved ejection fraction: Mirage or miracle? EMBO Mol. Med. 2020, 12, e10865. [CrossRef]

4. Zhou, S.G.; Ma, H.J.; Guo, Z.Y.; Zhang, W.; Yang, X. FHL2 participates in renal interstitial fibrosis by altering the phenotype of renal tubular epithelial cells via regulating the beta-catenin pathway. Eur. Rev. Med. Pharmacol. Sci. 2018, 22, $2734-2741$. [PubMed]

5. Tamura, N.; Ogawa, Y.; Chusho, H.; Nakamura, K.; Nakao, K.; Suda, M.; Kasahara, M.; Hashimoto, R.; Katsuura, G.; Mukoyama, M.; et al. Cardiac fibrosis in mice lacking brain natriuretic peptide. Proc. Natl. Acad. Sci. USA 2000, 97, 4239-4244. [CrossRef] [PubMed]

6. Hagler, M.A.; Hadley, T.M.; Zhang, H.; Mehra, K.; Roos, C.M.; Schaff, H.V.; Suri, R.M.; Miller, J.D. TGF-beta signalling and reactive oxygen species drive fibrosis and matrix remodelling in myxomatous mitral valves. Cardiovasc. Res. 2013, 99, 175-184. [CrossRef]

7. Iwaki, T.; Urano, T.; Umemura, K. PAI-1, progress in understanding the clinical problem and its aetiology. Br. J. Haematol. 2012, 157, 291-298. [CrossRef]

8. Valiente-Alandi, I.; Potter, S.J.; Salvador, A.M.; Schafer, A.E.; Schips, T.; Carrillo-Salinas, F.; Gibson, A.M.; Nieman, M.L.; Perkins, C.; Sargent, M.A.; et al. Inhibiting Fibronectin Attenuates Fibrosis and Improves Cardiac Function in a Model of Heart Failure. Circulation 2018, 138, 1236-1252. [CrossRef] [PubMed]

9. Muratoglu, S.C.; Belgrave, S.; Hampton, B.; Migliorini, M.; Coksaygan, T.; Chen, L.; Mikhailenko, I.; Strickland, D.K. LRP1 protects the vasculature by regulating levels of connective tissue growth factor and HtrA1. Arterioscler. Thromb. Vasc. Biol. 2013, 33, 2137-2146. [CrossRef] [PubMed]

10. Bayoumi, A.S.; Teoh, J.P.; Aonuma, T.; Yuan, Z.; Ruan, X.; Tang, Y.; Su, H.; Weintraub, N.L.; Kim, I.M. MicroRNA-532 protects the heart in acute myocardial infarction, and represses prss23, a positive regulator of endothelial-to-mesenchymal transition. Cardiovasc. Res. 2017, 113, 1603-1614. [CrossRef] [PubMed]

11. Sun, X.; Zhu, M.; Chen, X.; Jiang, X. MYH9 Inhibition Suppresses TGF-beta1-Stimulated Lung Fibroblast-to-Myofibroblast Differentiation. Front. Pharmacol. 2020, 11, 573524. [CrossRef] [PubMed]

12. Frangogiannis, N.G. The Extracellular Matrix in Ischemic and Nonischemic Heart Failure. Circ. Res. 2019, 125, 117-146. [CrossRef] [PubMed] 
13. Tsai, F.C.; Chang, G.J.; Lai, Y.J.; Chang, S.H.; Chen, W.J.; Yeh, Y.H. Ubiquitin Pathway Is Associated with Worsening Left Ventricle Function after Mitral Valve Repair: A Global Gene Expression Study. Int. J. Mol. Sci. 2020, 21, 5073. [CrossRef]

14. Vainio, L.E.; Szabo, Z.; Lin, R.; Ulvila, J.; Yrjola, R.; Alakoski, T.; Piuhola, J.; Koch, W.J.; Ruskoaho, H.; Fouse, S.D.; et al. Connective Tissue Growth Factor Inhibition Enhances Cardiac Repair and Limits Fibrosis After Myocardial Infarction. JACC Basic Transl. Sci. 2019, 4, 83-94. [CrossRef] [PubMed]

15. Montenegro-Venegas, C.; Tortosa, E.; Rosso, S.; Peretti, D.; Bollati, F.; Bisbal, M.; Jausoro, I.; Avila, J.; Caceres, A.; Gonzalez-Billault, C. MAP1B regulates axonal development by modulating Rho-GTPase Rac1 activity. Mol. Biol. Cell 2010, 21, 3518-3528. [CrossRef]

16. Giarratana, N.; Conti, F.; La Rovere, R.; Gijsbers, R.; Carai, P.; Duelen, R.; Vervliet, T.; Bultynck, G.; Ronzoni, F.; Piciotti, R.; et al. MICAL2 is essential for myogenic lineage commitment. Cell Death Dis. 2020, 11, 654. [CrossRef] [PubMed]

17. Brunskill, E.W.; Georgas, K.; Rumballe, B.; Little, M.H.; Potter, S.S. Defining the molecular character of the developing and adult kidney podocyte. PLoS ONE 2011, 6, e24640. [CrossRef]

18. Rabinovich-Nikitin, I.; Lieberman, B.; Martino, T.A.; Kirshenbaum, L.A. Circadian-Regulated Cell Death in Cardiovascular Diseases. Circulation 2019, 139, 965-980. [CrossRef]

19. Verkerk, A.O.; Lodder, E.M.; Wilders, R. Aquaporin Channels in the Heart-Physiology and Pathophysiology. Int. J. Mol. Sci. 2019, 20, 2039. [CrossRef]

20. Barutaut, M.; Fournier, P.; Peacock, W.F.; Evaristi, M.F.; Caubere, C.; Turkieh, A.; Desmoulin, F.; Eurlings, L.W.M.; van Wijk, S.; Rocca, H.B.; et al. Insulin-like Growth Factor Binding Protein 2 predicts mortality risk in heart failure. Int. J. Cardiol. 2020, 300, 245-251. [CrossRef]

21. Storey, J.D.; Taylor, J.E.; Siegmund, D. Strong control, conservative point estimation and simultaneous conservative consistency of false discovery rates: A unified approach. J. R. Stat. Soc. Ser. B Stat. Methodol. 2004, 66, 187-205. [CrossRef]

22. Yu, G.; Wang, L.G.; Han, Y.; He, Q.Y. clusterProfiler: An R package for comparing biological themes among gene clusters. OMICS J. Integr. Biol. 2012, 16, 284-287. [CrossRef] [PubMed]

23. Szklarczyk, D.; Franceschini, A.; Wyder, S.; Forslund, K.; Heller, D.; Huerta-Cepas, J.; Simonovic, M.; Roth, A.; Santos, A.; Tsafou, K.P.; et al. STRING v10: Protein-protein interaction networks, integrated over the tree of life. Nucleic Acids Res. 2015, 43, D447-D452. [CrossRef] [PubMed] 